\title{
Categorías identitarias espaciales para la comprensión de las controversias públicas: análisis de su empleo en Cataluña a propósito de los debates sobre el agua en España*
}

\author{
Spatial Identity Categories for the Understanding of Public \\ Controversies: Analyzing the Use of these Categories \\ in the Context of Water Debates in Spain
}

Recibido: mayo 16 de 2011 | Revisado: diciembre 13 de 2011 | Aceptado: julio 16 de 2012

\author{
JUAN C. ACEROS ** \\ José ANTONIO CAÑADA *** \\ MONTSERRAT RODRÍGUEZ \\ MIQUEL DOMÈNECH ***** \\ Universitat Autònoma de Barcelona, España
}

doi:10.11144/Javeriana.UPSY12-2.ciec

Para citar este artículo: Aceros, J. C., Cañada, J. A., Rodríguez, M. \& Domènech, M. (2013). Categorías identitarias espaciales para la comprensión de las controversias públicas: análisis de su empleo en Cataluña a propósito de los debates sobre el agua en España. Universitas Psychologica, 12 (2), 375-389.

* Naturaleza del artículo: investigación original. Este trabajo ha sido posible gracias al proyecto "La transformación de las controversias públicas en la sociedad del conocimiento. Un análisis psicosocial de la participación de expertos y ciudadanos en el debate sobre el agua", realizado en los años 2005 a 2008 con el apoyo de Ministerio de Educación y Ciencia de España (Ref. SEJ2005-09319-C03-01). Los autores desean expresar su agradecimiento a los participantes en los grupos de discusión y a los revisores anónimos de esta revista.

** Investigador en formación. Departament de Psicologia Social, Universitat Autònoma de Barcelona, España. Miembro del Grup d'Estudis Socials de la Ciència i la Tecnologia (GESCIT). E-mail: juancarlos.aceros@uab.es

**** Doctorando en la Universidad de Helsinki, Facultad de Ciencias Sociales, Departamento de Investigación Social, Unidad de Psicología Social. E-mail: jose.a.canada@helsinki.fi

${ }^{* * * * *}$ Profesor titular del Departament de Psicologia Social de la Universitat Autònoma de Barcelona. Actualmente es director del Grup d'Estudis Socials de la Ciència i la Tecnologia (GESCIT). E-mail: miquel.domenech@uab.cat

\section{RESUMEN}

Para acercarnos a cómo los ciudadanos catalanes dan cuenta de las controversias hídricas en España, se analizaron seis grupos de discusión realizados en Cataluña durante 2006. Se utilizó el concepto “identidad social espacial” para las interpretaciones, adaptándolo para resaltar su carácter interaccional. Al rastrear las categorías espaciales empleadas en las discusiones, se identificó la variedad de denominaciones usadas para debatir sobre los problemas hídricos en España. El análisis se centra en las denominaciones más usadas para determinar su utilidad e identificar las acciones discursivas que los participantes realizan con ellas. Los resultados indican que los hablantes dan cuenta de los "problemas del agua" movilizando una geografía política del sentido común que resalta el carácter socioespacial y conflictivo de los asuntos hídricos.

Palabras clave autores

Categorización, espacio, identidad espacial, identidad social, marcos interpretativos. Palabras clave descriptores

Análisis del discurso, investigación cualitativa, controversias sobre el agua.

\section{A B S T R A C T}

In order to know how Catalonian citizens account water controversies in Spain, we analyzed six focus groups carried out in Catalonia in 2006. A concept of 'urban social identity' is used for our interpretations, having been adapted to highlight its interactional character. Tracking the spatial categories used in the discussions, we identified the variety of denominations employed to debate water problems in Spain. The analysis is focused on the most frequent categories to establish their utility and identify discursive actions that participants produce. Results indicate that speakers account the "water problems" mobilizing a common sense political geography that highlights the socio-spatial and conflicting character of water issues.

Key words authors

Categorization, Space, Spatial Identity, Social Identity, Interpretation Frames.

Key words plus

Discourse Analysis, Qualitative Research, Water Controversies. 


\section{Introducción}

En las últimas décadas el agua se ha vuelto un tema de primer orden a nivel mundial. En España, el interés por los asuntos hidrológicos ha estado motivado por la protesta ambiental (Jiménez, 2005) que se ha presentado desde los años setenta (Bergua, 2000). Sin embargo, no es hasta los años noventa que la oposición al Plan Hidrológico Nacional y el trasvase del Ebro ${ }^{1}$ sitúan la cuestión como centro de un amplio interés político (Mairal, 1993). Este artículo es el resultado de una investigación interesada en conocer la percepción social de tal fenómeno. En dicha investigación, la identidad ligada al territorio demostró ser un factor a tener en cuenta (Aceros, Tirado \& Domènech, 2011). En las siguientes páginas nos acercamos a esta cuestión desde el concepto de "identidad social espacial" (Valera \& Pol, 1994).

Los datos provienen de seis grupos de discusión llevados a cabo en Cataluña (España). Su análisis se realiza en coherencia con el reciente giro discursivo en el estudio de la identidad espacial (Dixon \& Durrheim, 2000; Taylor, 2003) y está inspirado en el "espíritu etnometodológico" (Antaki \& Widdicombe, 1998). Concretamente, emplea herramientas analíticas provenientes del Membership Categorization Analysis (MCA). Con ellas se rastrea en el lenguaje en uso la utilización de denominaciones espaciales que sirven como dispositivos y categorías de identificación social. En este estudio se ofrece una aproximación a tres de las más frecuentemente encontradas en nuestros datos.

El artículo se organiza en cuatro apartados. Los dos primeros presentan consideraciones teóricas y metodológicas que introducen la forma como entendemos y estudiamos nuestro objeto de interés. El tercer y cuarto apartado presentan los resultados y la interpretación de los datos. En la discusión se resaltan las referencias espaciales más prominentes en los grupos de discusión ("España", "Cataluña" y "Valencia") y las

1 El trasvase del Ebro era una obra hidráulica contemplada en la Ley 10 de 5 de julio de 2001 del Plan Hidrológico Nacional y derogada en la ley 11/2005 de 22 de junio. Con ella se pretendía la transferencia de 1.050 hectómetros cúbicos anuales del río Ebro hasta las cuencas hidrológicas internas de Cataluña, del Júcar, del Segura y del sur de España. poblaciones adscritas a dichas categorías. El análisis identifica la movilización de una "geografía política del sentido común" que resalta el carácter socioespacial y conflictivo de los asuntos hídricos.

\section{La identidad social espacial, en la mente y en el discurso}

La relación entre identidad y espacio ha sido un tema de interés para diversas disciplinas. En psicología, aparece desde el trabajo de Fried (1963) sobre los efectos adversos de la relocalización forzada. Actualmente es un concepto central para la psicología ambiental (ver, Korpela, 1989; Lalli, 1992; Proshansky, Fabian \& Kaminoff, 1983) y para algunos psicólogos sociales (Dixon \& Durrheim, 2000; Taylor, 2003). En el contexto de habla hispana, Valera y Pol (1994) han destacado por su esfuerzo en redefinirlo desde la literatura sobre categorización social y comportamiento de grupos. Su aporte recibe el nombre de "identidad social urbana" (y, de manera más amplia, "identidad social espacial"), concepto que ha sido aplicado en varias investigaciones (ver, Uzzell, Pol \& Badenas, 2002; Valera, 1997; Valera, Guàrdia \& Pol, 1998).

La lectura que Valera y Pol (1994) hacen desde la perspectiva de la identidad social (Tajfel, 1981) y la Teoría de la Categorización del Yo (Turner, 1990) se ha vuelto popular en psicología en años recientes. Twigger-Ross y Uzzell (1996) proponen entender la identificación con los lugares como una expresión posible de la membresía a grupos humanos. Por su parte, Rollero y De Piccoli (2010) definen la dimensión social del apego de lugar a partir de la pertenencia a grupos territorialmente definidos. $\mathrm{Al}$ igual que estos autores, Valera y Pol (1994) conciben la identidad como un sistema cognitivo con el que la persona representa al self usando distintas "partes" de su "autoconcepto". Así, las personas son "perceptores" que cuentan con un conjunto estructurado de "identidades latentes" que se "activan" mentalmente en determinadas condiciones. Tales identidades toman la forma de categorías, es decir, de una "colección de estímulos" que aparecen como iguales al ser comparados con otra clase de "estímulos" (Turner, 1990). 
Las categorías identitarias, de acuerdo con este abordaje, se organizan jerárquicamente en tres niveles de abstracción (Turner, 1990): a) el nivel supraordenado que define la identidad de la persona como miembro de la especie humana, b) el nivel intermedio que la define a partir de la pertenencia grupal y c) el nivel subordinado que define a la persona como individuo específico y diferenciado del resto. Valera y Pol (1994) proponen que en el nivel intermedio se consideren categorías que indiquen la pertenencia a un entorno concreto (Uzzell et al., 2002; Valera \& Guàrdia, 2002). Estas categorías se organizarían en tres subniveles. El nivel con menor grado de inclusión sería el de "barrio". El siguiente nivel tendría un carácter inespecífico y se correspondería a la categoría "zona". Por encima de él aparecería la categoría "ciudad" como límite de lo que puede considerarse como una identidad propiamente "urbana".

Aunque Valera y Pol (1994) se muestran de acuerdo con el sistema cognitivo multinivel propuesto por la Teoría de la Categorización del Yo (TCY), también pretenden distanciarse del carácter individualista de la teoría y resaltar los elementos más propiamente sociales de la identidad (social) espacial. En este sentido, recurren al interaccionismo simbólico y al construccionismo social para defender que los entornos urbanos, entendidos como categorías, "vienen determinados por la interacción simbólica que se da entre las personas que comparten un determinado espacio y que se identifican con él a través de un conjunto de significados socialmente elaborados y compartidos" (Valera \& Pol, 1994, p. 11). De este modo, lo que inicialmente se entiende como parte del "autoconcepto" pasa a entenderse como una "construcción social". La identidad sigue siendo definida como el resultado de un proceso privado de categorización, pero los elementos con los que se estructura pasan a ser productos simbólicos de una "relación dialogante" (Valera \& Pol, 1994, p. 8) que individuos y grupos mantienen entre sí.

La necesidad de una comprensión más social de la identidad espacial ha sido apuntada por autores como Lalli (1992) o Lewicka (2008). Sin embargo, se han dado pocos pasos en el estudio de las "rela- ciones dialogantes" a las que se refieren Valera y Pol (1994). Los mismos autores, al usar predominantemente cuestionarios como técnica de recolección de datos, desatienden el carácter interactivo del fenómeno. Sin embargo, recientes avances en psicología discursiva han empezado a cambiar esta situación (Dixon \& Durrheim, 2000; Taylor, 2003). Desde la perspectiva discusiva, la identidad espacial es un acontecimiento intersubjetivo que ocurre durante el habla y gracias a ella. La construcción colectiva e interactiva de la identidad espacial ocurre mediante el uso de recursos simbólicos socialmente disponibles para los hablantes (entre ellos estarían las categorías, así como las metáforas, las ideologías, etc.) que se movilizan durante el intercambio discursivo para realizar diferentes acciones (como tomar posiciones, definir eventos, crear grupos, etc.). La identificación de los recursos usados y/o de las acciones realizadas es el objetivo del análisis discursivo de la identidad espacial.

En este trabajo se abordan los datos en coherencia con el giro discursivo en el estudio de la identidad espacial. Lo hacemos inspirados por una aproximación fundamentada en la sociología de Harold Garfinkel y en los trabajos de Harvey Sacks. De acuerdo con Antaki y Widdicombe (1998), el "espíritu etnometodológico" que caracteriza dicha aproximación se traduce en las siguientes ideas: 1) un hablante tiene una identidad cuando se le asigna o se asigna a sí mismo una categoría socialmente disponible, 2) el significado de esta adscripción reside en el contexto de su producción local, 3) el uso de la categoría vuelve relevante una identidad para la interacción en curso, 4) produciendo consecuencias en la interacción, 5) de una manera observable en la forma como los hablantes emplean el lenguaje en uso.

Actualmente, una de las aproximaciones metodológicas fundamentadas en el espíritu etnometodológico es el Membership Categorization Analysis (MCA). Dicha aproximación es un producto temprano de los trabajos realizados por Harvey Sacks sobre los métodos que los hablantes emplean para producir actividades como fenómenos observables y reportables (Silverman, 1998). El supuesto del que parte el MCA es que una de las formas de 
lograrlo es generando categorías que agrupan en "colecciones" lo que de otra manera serían "objetos" dispares (Antaki \& Widdicombe, 1998). Este proceso de categorización, a diferencia del propuesto por Turner (1990), no es una actividad mental sino una práctica social, metódicamente realizada en situaciones interactivas, a través del lenguaje. Concretamente, se trata de la selección y comprensión local y social de los términos más adecuados para referirse a los miembros de una población o grupo (Schegloff, 2007).

La categorización que interesa al MCA se lleva a cabo movilizando en la conversación tres tipos de recursos: los dispositivos de membresía por categorización (membership categorization devices), las categorías de membresía (membership categories) y las actividades ligadas a las categorías (category-bound activities). Las primeras son las denominaciones bajo las que se agrupan las segundas, es decir, son colecciones de categorías de membresía. Unas y otras tienen actividades o atributos convencionalmente asociados a ellas (Housley \& Fitzgerald, 2002); esto es, derechos y obligaciones imputables a los miembros de una población dada (Schegloff, 2007). La vinculación recurrente-de "sentido común"-entre los dispositivos, las categorías y las actividades hace que los hablantes puedan derivar los unos de los otros, aplicando una serie de reglas.

El MCA se ha utilizado predominantemente para el estudio de las categorías descriptivas aplicables a los seres humanos (Housley \& Fitzgerald, 2002), entre las que se encuentran los gentilicios (Schegloff, 2007). También se ha utilizado para analizar la selección de formulaciones de localización relevantes (Shegloff, 1972) y lo que McHoul y Watson (1984) llaman las "ecologías del sentido común", es decir, las maneras profanas de organización del conocimiento geográfico. Nosotros proponemos trasladar esta aproximación metodológica al estudio de las denominaciones o referencias espaciales que se vuelven relevantes en contextos de interacción y que funcionan como dispositivos de identificación de personas y grupos. Se trata de hacer, desde un punto de vista discursivo, lo que Valera y Pol (1994) proponen desde una mirada predominantemente cognitiva.

\section{Método}

\section{Material}

Los datos provienen de seis grupos de discusión realizados en diferentes ciudades catalanas (Amposta, Barcelona, Girona, Granollers, Tarragona y Terrassa) durante el año 2006. Este tipo de metodología se utiliza actualmente como una de las formas de obtener datos relevantes para la investigación etnometodológica (ver, Puchta \& Potter, 2004). Los grupos tenían una composición heterogénea, pero estaban organizados en torno a los mismos criterios. Se procuró un número igual de hombres y mujeres, con diferentes rangos de edad (jóvenes, adultos y personas mayores) y un perfil ocupacional variado. Las ciudades seleccionadas representan dos tipos de proximidad geográfica con respecto al principal foco de conflicto en relación al trasvase del Ebro. Mientras Amposta y Tarragona son poblaciones cercanas al río Ebro; Barcelona, Girona, Granollers y Terrassa, son geográficamente más distantes. Entre las poblaciones seleccionadas, tres de ellas son capitales de provincia (Barcelona, Tarragona y Girona) y tres son capitales de comarca (Amposta, Granollers, Terrassa). Las poblaciones se diferencian, entre otras razones, por su población, siendo Amposta la menos poblada (21.558 habitantes), seguida de Granollers (60.000 habitantes) y Girona (96.722 habitantes). Barcelona es la ciudad más poblada (1.619.337 habitantes), mientras que Tarragona (130.323 habitantes) y Terrassa (212.724 habitantes), tienen una población intermedia.

\section{Participantes}

Como es bien sabido, la representatividad estadística no es una cuestión relevante en las investigaciones basadas en una aproximación cualitativa. En este trabajo se empleó un muestreo propositivo (Vaughn, Schumm \& Sinagub, 1996), que asegura la necesaria diversidad de los participantes y que nos proporciona una muestra estructurada antes que aleatoria. En este sentido, se desarrolló una estrategia de muestreo basada en el tema a tratar. Básicamente, se procuró asegurar que entre las 
personas participantes hubiera diferentes procedencias geográficas, así como diferentes percepciones del tema en cuestión. Se procuró, también, que las personas participantes en un grupo de discusión no se conocieran previamente.

Se contó con la participación de un total de 45 personas. Cada grupo contó con un promedio de 8 participantes. La mayor parte eran jóvenes y adultos, con una edad promedio de 44 años. Todos eran de nacionalidad española. Su distribución en los diferentes grupos obedecía a su lugar de residencia habitual. Los participantes tenían perfiles ocupacionales diversos, con lo que se contó con la presencia de estudiantes universitarios, profesores e investigadores, funcionarios públicos, psicólogos, empleados del sector bancario, empleados del tercer sector, médicos, ingenieros, periodistas, trabajadores del hogar, vendedores, jubilados y personas en paro. La mayor parte de los asistentes no manifestaba ningún conocimiento especializado en la temática (salvo algunos miembros de grupos concernidos o voluntarios en programas ambientales). De ellos no se esperaba un posicionamiento experto. Su saber espontáneo sobre los problemas del agua en España era suficiente para participar. El hecho de que las movilizaciones contra el trasvase del Ebro fueran cercanas hacía de la cuestión un tema de conversación reconocible para todos ellos.

\section{Procedimiento}

Al principio de cada grupo se presentaban dos anuncios de periódico que manifestaban posiciones distintas respecto al trasvase del Ebro. A partir de las primeras intervenciones, la conversación fluía de manera espontánea. Con el fin de orientarla hacia los objetivos de la investigación, el moderador manifestaba su interés por conocer la opinión de los asistentes sobre temas como el papel de expertos, políticos y ciudadanos en la controversia, las distintas formas de participación de la sociedad civil, o los medios a través de los cuales las personas se informan sobre el debate. Aunque ninguna de las preguntas formuladas por el moderador apuntaba intencionalmente a la identidad espacial, pronto resultó evidente que la adscripción territorial era un recurso invocado para articular opiniones al respecto de las controversias sobre el agua.

El desarrollo de las discusiones se grabó en audio con la autorización de los participantes. La información recolectada se sometió a una transcripción basada en el contenido. Las transcripciones fueron leídas repetida e independientemente por todos los investigadores con el objetivo de identificar referencias espaciales explícitas. Las referencias encontradas se introdujeron en un fichero informático, ordenadas en un continuo que iba desde las de orden general (supraordenadas) a las menos inclusivas (subordinadas). Cada denominación espacial encontrada se introducía en el fichero junto con los turnos anteriores o posteriores. Con los datos así organizados, fue posible 1) encontrar las referencias más utilizadas en nuestros grupos, 2) centrar el análisis en tales referencias y 3) recuperar, para cada una, su contexto más inmediato de enunciación.

\section{Resultados}

A partir del tratamiento de los datos, se encontraron 71 tipos de referencias espaciales empleadas en los grupos de discusión. Fueron identificadas tanto divisiones administrativas (nombres de países o ciudades), como nombres de zonas y territorios (como "Tierras del Ebro" ${ }^{2}$ ), y lugares genéricos como "casa" o "campo de golf". Cada uno de estos tipos de referencias espaciales incluye diferentes denominaciones que pueden organizarse jerárquicamente, siguiendo una lógica concéntrica (Shegloff, 1972). Una representación formal de dicha organización puede verse en la Figura 1. Allí se muestra una versión simplificada de las principales jerarquías que pueden confeccionarse con los datos. Dos de ellas son taxonomías del tipo todo-parte (partonomies, en términos de Bilmes, 2009) y son identificables por sus relaciones en líneas continuas. La primera organiza las divisiones administrativas (con "Europa" como categoría superordenada); la otra hace

2 Se denomina "Tierras del Ebro" a un conjunto de comarcas ubicadas al sur de Cataluña y que tienen en común el paso del curso bajo del río Ebro por su territorio. 


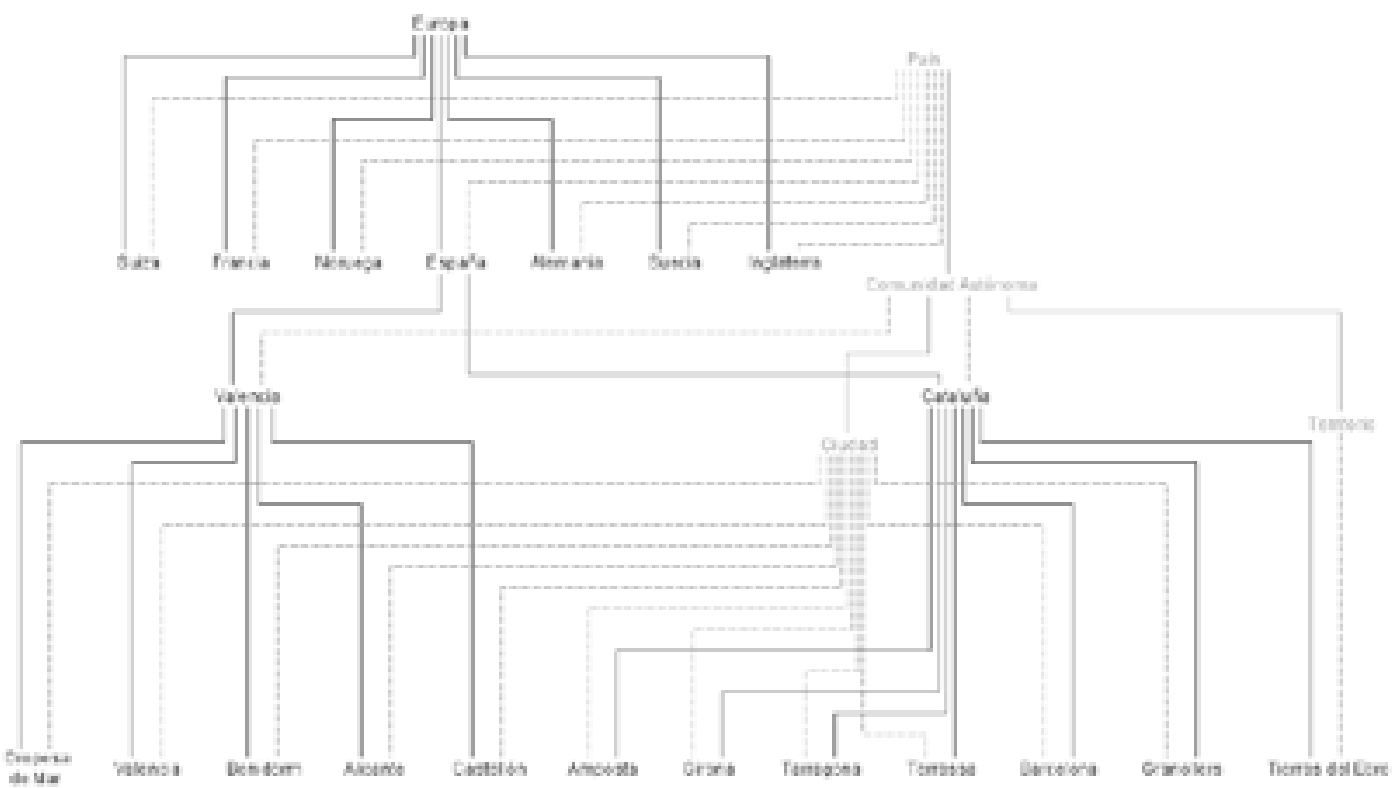

Figura 1. Organización de los datos en jerarquías, versión simplificada.

Fuente: elaboración propia.

lo propio con tipos de áreas geográficas (con "país" a la cabeza). La tercera es una taxonomía de inclusión cuyas relaciones se representan con líneas punteadas, indicando por ejemplo tipos de países o de comunidades autónomas.

Estas taxonomías formalizan la ecología del sentido común rastreada. Como puede apreciarse, la misma incluye denominaciones muy variadas. Con el fin de reconocer tal riqueza, y en la línea de otros trabajos que apuntan la naturaleza multinivel de la identidad espacial (Altman \& Low, 1992; Hidalgo \& Hernández, 2001; Lalli, 1992), nuestro análisis no se limitó a examinar el uso discursivo de las referencias "urbanas". Esto implicó que el "barrio", la "zona" y la "ciudad" perdieran la importancia que les otorgan Valera y Pol (1994). A continuación, se ofrece una interpretación del uso discursivo de referencias con mayor nivel de generalidad. Nos centramos en tres de las empleadas con mayor frecuencia: "España", "Cataluña" y "Valencia". El examen de otros topónimos y etiquetas recurrentes -como "país", "Tarragona", "Tierras del Ebro", y "campos de golf"- se pospone para posteriores trabajos.

\section{Análisis}

España: Territorio, población y gobierno en déficit (...) ahora hablemos a nivel de país. España, ieh? Tenemos un, un problema de agua. (Granollers, H1. 16)

Dentro de la taxonomía de divisiones administrativas que hemos construido a partir de los datos, "España" ocupa un lugar intermedio. Sin embargo, en la práctica discursiva opera como una categoría superordenada o, más exactamente, como un dispositivo de membresía. Al activar la referencia a "España" los participantes movilizan un dispositivo de categorización por localización (McHoul \& Watson, 1984) que define la situación sobre la que conversan y vuelve reconocibles una serie de "problemas del agua", particularmente referidos a la escasez del recurso. Esto se logra vinculando a "España" predicados como "árido" o "seco", y utilizando tales predicados en comparaciones entre "España" y otros países europeos - de los que se dice que gozan de lluvias abundantes (Granollers, H1. 7) y de una gran cantidad de recursos hídricos (Amposta, H3. 15)-, así como entre la llamada "España 
Fragmento 1, Granollers

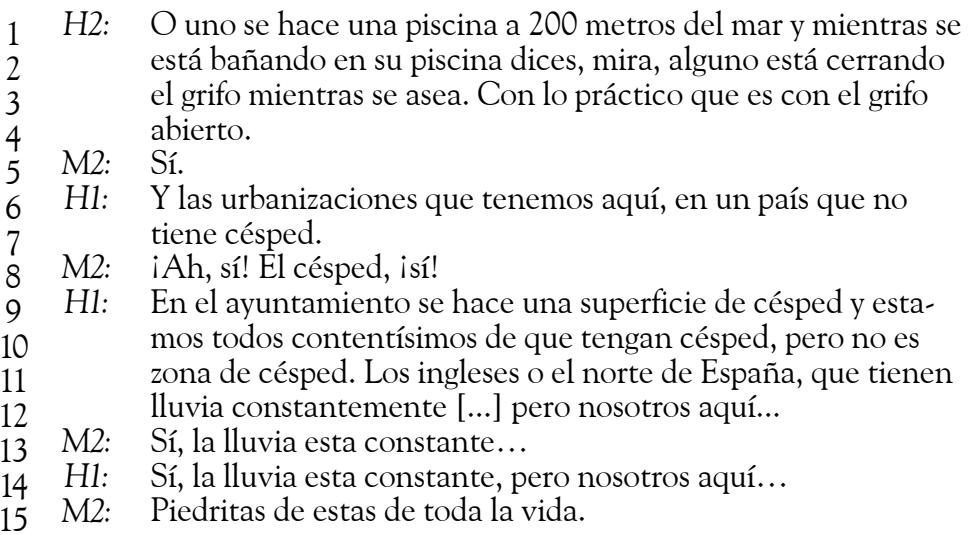

seca" (al sur y al este) y una "España húmeda" (al noroeste). Es a través de este tipo de caracterizaciones y comparaciones que "España" se vuelve una categoría relevante para hablar de los "problemas del agua". El siguiente fragmento es un ejemplo de cómo ocurre en los grupos de discusión.

Este fragmento forma parte de una secuencia en la que los participantes examinan colectivamente los usos del agua en distintos lugares. Al hacerlo están asignando valores a determinadas actividades. Controlar el gasto doméstico de agua, aunque es poco "práctico" (línea 3), es preferible a tener "una piscina a 200 metros del mar" (línea 1). Dicha preferencia se sostiene sobre un diagnóstico común de la situación: "España", en comparación con "Inglaterra", es un territorio con un déficit hídrico, es un territorio "seco" (como lo es el sur de España, en comparación con el norte). Este marco de escasez hace aceptables determinados usos del agua, mientras que vuelve reprobables otros. Así pues, el dispositivo no simplemente dibuja un estado de cosas, sino que permite a lo hablantes derivar cursos de acción válidos o inválidos. Sugiere cómo debe comportarse una población dada ("nosotros aquí", en la línea 14).

En tanto que dispositivo de categorización, "España" -y más concretamente "España seca"-, tiene asociadas unas categorías y unas actividades. Adicionalmente, sirve para agrupar e identificar a una o varias poblaciones. Un examen de los usos del agua consistentes o inconsistentes con la "España seca" puede ayudarnos a identificar dichas pobla- ciones. La construcción de "superficies de césped" (suponemos, más allá de ciertos límites) o de "una piscina a 200 metros del mar" serían ejemplos de actividades inconsistentes con la "España seca"; mientras que construir superficies cubiertas de grava (las "piedritas de toda la vida") y controlar el uso doméstico del recurso serían consistentes. Dichas acciones son imputables a los "consumidores" o "usuarios finales" y a las "administraciones" o "Gobiernos". Tales grupos están convencionalmente vinculados en el discurso: son pares normalmente relacionados (Silverman, 1998). Para clarificar el tipo de relación que se les adjudica, podemos examinar el fragmento 2 .

En el fragmento 2, los hablantes conversan sobre lo que el primero de ellos denomina la "cultura del ahorro". Aunque dentro de dicha conversación se inserta una secuencia sobre el costo del recurso (líneas 3 a 11), de lo que se trata es de describir la posición de los españoles ante los "problemas del agua". El primer hablante (H1) deja claro que "el ciudadano no tiene una cultura del ahorro". A dicha formulación le sigue una exculpación. El despilfarrador de agua se construye como un personaje nuevo en la sociedad española. Solo en tiempos recientes se ha vuelto un problema: lo es "ahora" porque el agua se ha convertido en un asunto de interés público ("se habla de desalinizar") y porque la penalización de su comportamiento "abusivo" se plantea en la actualidad.

La caracterización del ciudadano español como una persona "sin cultura de ahorro" es apoyada por 
Fragmento 2, Terrassa

$1 \quad$ H1: También hay otra cuestión que es el ciudadano no tiene una cultura del ahorro. El agua siempre se ha visto como una cosa que cuesta poco, ino? (...) Ahora ya se habla de desalinizar y de que el uso abusivo se debe penalizar.

M1: Porque los recibos están subiendo (...) yo tengo recibos que se han triplicado.

H1: Aquí el consumidor paga el agua 5 veces más cara que en Murcia.

M1: Pero es que pagamos todo más caro que en Murcia.

H2: $\quad$ En cambio el IPC [Índice de Precios al Consumidor] que pagamos es el mismo (...) Pero yo como consumidor, por ejemplo, pues puede ser que haya gente que en el lavabo, en el depósito del lavabo, pues tienen una botella y unas cosas que venden para que no se tenga que tirar siempre de la cadena o toda la cisterna. Pues eso muy poca gente lo tiene en este momento: tira la cadena y si luego el otro vuelve a tirar la cadena, y vuelve a tirar la cadena...

M1: ... y cuando nos duchamos, abrimos el grifo para que se vaya calentando y te estás quitando la ropa antes de meterte, y así la mayoría de la gente.

H2: Ni menos, ni más. Pues eso es una falta de cultura de agua que ya en la escuela, desde pequeños, ya se nos debería enseñar, y los padres deberían comenzar a pensar que a la larga no tendremos agua ni para beber, ino? Esta pedagogía del ahorro del agua tampoco se ha hecho un plan nacional. El Gobierno tampoco se ha preocupado de concienciar las diferentes instituciones educativas, familiares y tal.

los demás hablantes. $\mathrm{H} 2$ sostiene que "muy poca gente" usa tecnologías ahorradoras; mientras que M1 afirma que "la mayoría de la gente" no controla su consumo doméstico. La exculpación del "consumidor" también es co-producida. Esto es particularmente claro en el último turno de $\mathrm{H} 2$ en el que se explica la falta de "cultura de ahorro" por la ausencia de un "plan nacional" que implemente una "pedagogía del ahorro". El par consumidor, Gobierno, es especialmente visible aquí. Si los ciudadanos españoles actúan de manera ineficiente en la "España seca" es porque el "Gobierno" no regula su conducta. Dicha situación es valorada implícitamente como inapropiada; es decir, como una desviación de las actividades asociadas a la categoría "Gobierno", del que se espera un papel activo en los asuntos de interés público.

Como lo enuncia uno de los participantes del grupo de Terrassa: “(...) estamos siempre en una visión de país en la que todo tiene que surgir de la administración" (Terrassa, H2. 18). Sin embargo, para el caso de la "pedagogía del ahorro", el papel activo del "Gobierno" no termina por desplegarse y, en consecuencia, se justifica que los "ciudadanos" asuman un papel pasivo en el control del gasto de agua. El comportamiento de los segundos aparece como determinado por la acción de los primeros. Al formularse de esta manera, el par gobiernoconsumidores complementa en el campo sociopolítico lo que en principio se había descrito como una interpretación de la situación hidrológica. Los "problemas del agua" no tienen solo la "aridez" de "España" por fundamento, sino la pasividad de la población. "España" es entonces, en el contexto de los fragmentos examinados, un dispositivo con el que se puede dar cuenta de un triple déficit: el de un territorio "seco" (línea 24), el de un Gobierno desinteresado por el control de la demanda (línea 25) y el de un conjunto de usuarios "no concienciados" (línea 26).

En resumen, movilizar la categoría "España” en el contexto de conversaciones sobre las controversias hídricas permite a los hablantes: 1) definir una situación problemática, 2) ubicar a una población en el marco de dicha situación y 3) delimitar acciones aceptables o inaceptables para esta población. Las dos últimas acciones discursivas, en tanto que movilizan categorías grupales y hacen imputables 
ciertos derechos y obligaciones, son las más directamente implicadas en procesos de identificación. Pero las identidades resultantes son al mismo tiempo sociales (referidas a grupos humanos como el "Gobierno" o los "consumidores") y espaciales (delimitadas geográficamente por su membresía a "España"); son identidades híbridas (Darier \& Tàbara, 2006). No se trata de gobiernos o consumidores en general, sino de las administraciones y los usuarios "españoles". Así pues, para reconocer y describir los "problemas del agua", los hablantes no solo movilizan una geografía o una sociología profana, sino una geografía política del sentido común. Para profundizar en cómo dicha geografía política profana sirve a los hablantes para dar sentido a situaciones dilemáticas -como los "problemas del agua"-, se examina a continuación el uso que hacen de las denominaciones "Cataluña" y "Valencia".

"Cataluña" vs. "Valencia": La geografía política profana de un conflicto

(...) Si los valencianos vienen y nos lo colocan, nosotros como catalanes nos defenderemos, ino? (Granollers, H2. 4)

Apelar a una geografía política del sentido común puede ser de utilidad para ofrecer una comprensión más social de la identidad espacial. Lo es en la medida en que se entienda, no como un conjunto de cogniciones relativamente organizadas en la interioridad del sujeto, sino como una forma de conocimiento en acción (Housley \& Fitzgerald, 2002) con el que se otorga colectivamente significado a, y se da cuenta de, los "problemas del agua". En este sentido, se propone entender dicha geografía mundana como un conjunto de recursos interpretativos empleados por los hablantes para organizar socialmente el espacio (Housley \& Smith, 2011), y que se expresan a través de un conjunto de formulaciones socio-político-espaciales efectivamente activadas y movilizadas en el lenguaje en uso.

En un trabajo sobre las audiencias públicas a propósito de la violencia en Irlanda del Norte, en 1969, Drew (1978) documenta cómo los testigos producen un recuento de los acontecimientos con atención a la distribución de los grupos religiosos en el espacio. Tal "organización religiosa de la geografía de Belfast" (p. 4) no es inocente, afirma el autor, sino que forma parte de los métodos a través de los cuales es posible formular acusaciones. La geografía mundana que hemos encontrado en nuestros datos se presta para una lectura similar. La invocación interactiva de determinados dispositivos y categorías espaciales sirve para dar cuenta de la distribución de las posiciones políticas en las controversias sobre el agua, así como para juzgar la conducta de determinadas poblaciones. La forma como los hablantes construyen -hablan del "conflicto entre comunidades autónomas"- es particularmente ilustrativa a este respecto.

Las comunidades autónomas son entidades territoriales recogidas en la Constitución Española de 1978. Desde 1995, el Estado español se divide política y administrativamente en diecisiete comunidades autónomas con sus propios órganos de gobierno, instituciones representativas y competencias ejecutivas. En los grupos focales se hizo mención de algunas de ellas: Andalucía, Aragón, Castilla-La Mancha, Cataluña, Islas Baleares, Murcia y Valencia. Aunque todas son útiles para hablar sobre los "problemas del agua", Cataluña y Valencia sobresalen por ser las más utilizadas. Las dos tienen un papel central en la comprensión social de las controversias sobre el agua, ya que permiten a los hablantes la producción de opiniones reconocibles y aceptables en el contexto de realización de los grupos de discusión. En dichas opiniones, los contenciosos se reducen a un enfrentamiento entre dos entidades discretas: "Valencia está diciendo [trasvase del] agua sí, y Cataluña y Aragón dicen agua no" (Granollers, N2. 19). Más adelante se volverá sobre el particular, resaltando una forma de problematizar dicha simplificación. En esta sección interesa resaltar cómo la movilización de "Cataluña" y "Valencia" facilita la toma de posición en asunto cuyos detalles técnicos son de alta complejidad. Para tal fin, se examina el fragmento 3 , un extracto monológico que aparece como respuesta a una conversación sobre la política de aguas.

Con anterioridad a este fragmento, los hablantes articulan su desconfianza ante los expertos y políticos que participan en la gestión del agua en España. 
Fragmento 3, Girona

1 H2: A pesar de lo que decíamos no pierdo la esperanza. Mira, ayer veía un acto que me parece una tontería integral, que es decidir quién es el catalán o la catalana del año (...) Iba mirando así, de reojo, y dije: bueno, aún es un pueblo sano (...) Un pueblo que reconoce las trayectorias personales, la lucha personal, el esfuerzo. Por tanto, dije, no desconfío más de nosotros mismos, ¿no? (...) Además va a echar un discurso diciendo: este premio no lo recojo en nombre propio, sino que lo recojo en recuerdo de todos los hombres y mujeres que lucharon por la República. (...) Y bueno, eso, que yo creo que la gente tenemos mucho que decir, ino? Y que encontraremos maneras y que no somos tan tontos como a veces los que nos gestionan se piensan. $Y$ creo que hay diferencias de percepción... a nivel español creo que se hace un tipo de política que no congenia mucho, por lo que sea ieh? Historia, diferentes grupos, maneras de entender el territorio, la sociedad que hay en Cataluña. Yo creo que es muy diferente y eso a veces provoca dicotomías.

H2 se posiciona frente a tal opinión, desvinculando a la población catalana ("un pueblo sano", línea 4) del comportamiento de las administraciones ("los que nos gestionan”, línea 12). En su intervención "Cataluña” cobra sentido dentro del dispositivo "España", por oposición al "tipo de política" (línea 14) desarrollada a nivel estatal. La alusión a la "catalana del año" que dedica el galardón a quienes "lucharon por la República" sirve de antesala a dicha caracterización. Al enmarcar su intervención con esta referencia, la orientación política se activa como un criterio para distinguir entre "Cataluña" y "España”. Concretamente, el republicanismo de izquierdas queda asociado a "Cataluña", mientras que la derecha política queda implícitamente vinculada a "España"

Este sencillo mapa político no solamente distingue a "Cataluña" de "España”. A la distribución

3 Este fenómeno se puede interpretar como la generación discursiva de distintividad para la categoría "Cataluña". En otros grupos de discusión, dicho proceso se aprecia en relación con los temas hidrológicos cuando se llama la atención sobre el enlace entre la identidad catalana y el río Ebro. Su paso por "Cataluña" es valorado por los hablantes como un fenómeno positivo (Granollers, H4. 16), articulador de las llamadas "Tierras del Ebro" (Amposta, D3. 31). Adicionalmente, los participantes describen su comunidad autónoma como un lugar donde se hace un mejor uso del agua que en otros territorios: "Hay lugares, no aquí en Cataluña, (...) que hay más piscinas que pueblo" (Granollers, H2. 8). Se distancian así de la falta de "cultura de ahorro" que atribuyen al resto de "españoles"; se distinguen de ellos resaltando los atributos positivos de su categoría y estableciendo el Ebro como objeto de mutua identificación. espacial de diferentes "formas de hacer política" se atribuye la "generación de dicotomías" (línea, 17). Esta formulación se aplica en el fragmento a "España", pero también puede servir para hablar de otras categorías espaciales agrupables bajo dicho dispositivo de membresía. El distanciamiento político entre los catalanes y otras comunidades autónomas suele construirse alrededor de lo que los hablantes llaman "anti-catalanismo". Se trata de una acción -emprendida por miembros de otras categorías del mismo nivel de generalización- consistente en describir a los "catalanes" con predicados del tipo "egoístas" (Barcelona, N1. 3), "bordes" (Girona, N2. 5) o "insolidarios" (Amposta, D3. 5). La categoría "Valencia" fue una de aquellas a las que los interlocutores atribuyeron tal anti-catalanismo.

La construcción que los hablantes hacen de la referencia a "Valencia" se asemeja a la que hemos descrito a propósito de la "España seca". Los participantes caracterizan a "Valencia" por su "clima seco" (Granollers, H3. 14) que le da al territorio el aspecto de un "desierto total” (Granollers, H3. 15). Mientras que en "Cataluña" reconocen el paso del río Ebro, los hablantes llaman la atención sobre el carácter deficitario de los ríos alóctonos de la vecina comunidad autónoma -"el Júcar, que es el río del País Valenciano llega, pues, prácticamente sin agua" (Terrassa, H1. 2)-, así como sobre el peligro que corren sus ecosistemas acuáticos -"La albufera desaparecerá, porque el agua la están chupando en 
Castilla-La Mancha” (Terrassa, H1. 3)-. Aunque "Cataluña" forma parte de la "España seca", con respecto a "Valencia" se presenta como más privilegiada en términos hidrológicos. Este contraste entre lo seco/ajeno y lo húmedo/propio ha sido documentado por Mairal (1993) como una forma que tienen los afectados por infraestructuras hidráulicas de expresar el apego a la propia tierra. Aquí aparece como una estrategia discursiva que sirve para dibujar un contexto político, de conflicto intergrupal, y para asumir una posición a favor de una categoría de la que los hablantes son miembros: "Cataluña".

El contencioso entre "Cataluña" y "Valencia" se co-produce discursivamente en los grupos de discusión a través de conversaciones sobre el Plan Hidrológico Nacional: "Este plan hidrológico", dice uno de los participantes, "es un intento de enfrentar Cataluña con el País Valenciano” (Terrassa, H1. 3). El enfrentamiento tendría como eje el trasvase del río Ebro -apoyado por "Valencia" y rechazado por "Cataluña" - y estaría originado por los usos del recurso que se dan en el territorio valenciano: "Es como se gestiona el agua en Valencia lo que lleva a estos conflictos" (Girona, N2. 2). El significado de dicha gestión se construye con referencias a un conjunto de actividades "insostenibles" asociadas: la construcción desmedida de complejos turísticos (Amposta, H3. 14, Tarragona, H2. 9), "campos de golf" (Barcelona, M2. 3, Barcelona, H1. 4, Tarra- gona, M4. 6), "bloques de pisos" (Granollers, H2. 1), "rascacielos" (Granollers, H4. 18) o "segundas residencias" (Barcelona, D2. 3; Granollers, H2. 18). En otras palabras, los "problemas del agua" en los que están implicados "Cataluña" y "Valencia" no se atribuyen solo a las condiciones ambientales, sino además (y en ocasiones, exclusivamente) al comportamiento del "Gobierno" valenciano. En la siguiente sección se profundiza en la manera como dicha atribución ayuda a los hablantes a comprender la identidad espacial valenciana, interpretando el "enfrentamiento entre comunidades" en el producto de una maquinación política.

Las identidades espaciales como herramienta política

(...) con la Generalitat Valenciana está clarísimo: es demagogia política, ino? Es un poco más la historia de (...) la utilización, en este caso, del recurso del agua (...) como enfrentamiento entre comunidades. (Barcelona, N1. 1)

En los grupos de discusión examinados, los hablantes co-producen discursivamente los conflictos sobre el agua en términos que para ellos son reconocibles. Para hacerlo, despliegan elementos de lo que hemos llamado la geografía política del sentido común; esto es, conocimientos compartidos sobre las interacciones entre el territorio y sus formas de

Fragmento 4, Granollers
H2: Yo básicamente porque tengo poca información, pero básicamente yo supongo que es montar un canal aquí (...) y llevarlo para allá. Y lo que he escuchado (...) por los medios y tal es que (...) si el Ebro está vacío, ¿no? No agarramos agua. $\mathrm{O}$ sea, solo coger agua si hay agua [en el Ebro]. Yo eso por mucho que me lo expliquen, no me lo creo. Porque he visto ejemplos de que no lo hacen. $\mathrm{O}$ sea, si les dejas harán lo que quieran (...)
M2: Pero a mí lo que me da rabia...
H2: ... harán lo que quieran...
M2: ... es que han conseguido lo que querían, que era politi- zar el tema.
H2: iSí!
M2: Porque usted mismo acaba de decir, si les dejas poner...
H2: Sí
M2: ... como una cuestión política, es decir, si los valencia- nos llegan y nos lo colocan, nosotros como catalanes nos defenderemos, ino? Ya van al tema este, ino? Valencia, Cataluña, Cataluña, Valencia.


ordenamiento. Dicho ordenamiento puede ser caracterizado de muchas maneras. En los fragmentos analizados, los hablantes producen sus opiniones utilizando referencias de orden político. En esta sección se profundiza en una de las implicaciones de esta forma de hacer reconocibles los "problemas del agua" en España: la formulación de las controversias hídricas como situaciones conflictivas. Para tal efecto, se examina el siguiente fragmento.

En este fragmento los "problemas del agua" aparecen como una "cuestión política". Esto ocurre en al menos dos sentidos. En primer lugar, presentando el trasvase del Ebro ("montar un canal aquî", línea 2) como una acción asociada a los políticos (líneas 6-8). En segunda instancia, hablando del enfrentamiento entre "Valencia" y "Cataluña" como el producto de una "politización" (líneas 11-12). Ambas cuestiones están relacionadas: M2 produce su opinión sobre la "politización" de los contenciosos hídricos tomando como punto de partida la descripción que $\mathrm{H} 2$ hace del comportamiento esperable de los políticos "valencianos" (línea 14). El conflicto entre comunidades autónomas queda entonces planteado como parte de los esfuerzos que los "políticos" realizan para configurar un determinado estado de cosas, delimitar las acciones posibles y asignar responsabilidades ("si los valencianos llegan y nos lo colocan, nosotros como catalanes nos defenderemos", líneas 16-18).

Como hemos visto en este trabajo, tal forma de emplear las identidades espaciales no es una actividad exclusiva de quienes ocupan posiciones de poder. Los mismos hablantes participan de esta "politización". Al visibilizar la dimensión a la vez geográfica y política de la identidad, los hablantes hacen también política identitaria; esto es, construyen los asuntos hídricos como cuestiones problemáticas y conflictivas, donde distintos grupos se adjudican a sí mismos, y le asignan a otros, determinadas categorías asociadas a ciertos derechos y obligaciones. La movilización de referencias a las comunidades autónomas sirve en este sentido para darle forma a un contencioso 1) delimitando la propia pertenencia grupal de los hablantes, 2) distinguiéndolos de otros grupos, 3) haciendo reconocible un conflicto entre las poblaciones territorialmente definidas y 4) relativizando la base misma de dicho conflicto, esto es, denunciando la "manipulación" estratégica de las categorías espaciales.

La "manipulación" estratégica de la identidad espacial es un tema central en la geografía política del sentido común. En los grupos de discusión aparece en repetidas ocasiones como una forma de dar cuenta de la dimensión política de las categorías espaciales. Si bien esto puede ocurrir tanto para el caso de "Cataluña" como para el de "Valencia", las referencias a dicha "manipulación" revelan una posición a favor del grupo propio y se asocian con más frecuencia al exogrupo. Un participante del grupo de discusión de Barcelona expresa con especial claridad dicha adscripción cuando describe la situación de los "valencianos” así: “...hay gente que realmente, o sea, la ignorancia es absoluta y han llegado a ir a manifestaciones convocadas en $\mathrm{Va}$ lencia, pagadas por la Generalitat Valenciana con dinero público (...) defendiendo lo que es el agua del río. Eso es vergonzoso porque realmente de lo que se trata es de una manipulación de todo un tema" (Barcelona, N1. 3). Aparece aquí de nuevo el par Gobierno-usuarios con el que los "valencianos" son caracterizados como actores ingenuos, movilizados por motivos que no son los suyos propios. Su participación en manifestaciones a favor del trasvase del Ebro tiene como origen la acción de "los políticos" y, específicamente, la "Generalitat Valenciana".

Las referencias al uso estratégico del par Cataluña-Valencia es parte de los métodos a través de los cuales los hablantes dibujan los contornos políticos de los "problemas del agua". A través de dicha práctica, dan cuenta de las identidades espaciales como logros o productos sociales. Así pues, en los grupos de discusión los participantes no solamente vuelven relevantes ciertas categorías, sino que pueden llegar a reconocer su carácter artificial e, incluso, artificioso. Por esta vía, llaman la atención sobre el papel que cumple la identidad en las luchas históricas entre grupos (Reicher, 1996) y sobre la dimensión política que tienen dichas luchas. Profundizar en esta cuestión, poco explorada en la literatura sobre identidad espacial (Dixon \& Durrheim, 2000), puede abrir vías de estudio en el futuro. 


\section{Conclusiones}

Con el objetivo de acercarnos a cómo los ciudadanos catalanes dan cuenta de las controversias hídricas en España, en este artículo se han analizado seis grupos de discusión realizados en Cataluña (España). Una primera aproximación a los datos ha permitido rastrear un conjunto de denominaciones espaciales que los participantes utilizan cuando hablan sobre los "problemas del agua". El análisis se ha centrado en las tres categorías más frecuentemente utilizadas: "España", "Cataluña” y "Valencia”. Estas etiquetas se han interpretado como herramientas discursivas (dispositivos y categorías de membresía) con las que los hablantes co-producen identidades espaciales reconocibles a través de la lengua en uso.

El análisis indica que los participantes movilizan las denominaciones "España", "Cataluña" y "Valencia" para llevar a cabo diferentes acciones discursivas. Una de ellas tiene que ver con la identificación de poblaciones cuyas actividades se consideran relevantes para abordar y entender los "problemas del agua". Dichas poblaciones han aparecido en la forma de dos diferentes pares normalmente relacionados. Uno de ellos está integrado por el "Gobierno" y los "usuarios finales", e implica un vínculo de dependencia del segundo grupo con respecto del primero. El otro par está compuesto por dos comunidades autónomas enfrentadas por el trasvase del Ebro, y se describe como el resultado de una maquinación urdida para favorecer intereses económicos y partidistas.

La activación de las dos parejas antes mencionadas construye los "problemas del agua" como asuntos políticos además de geográficos, y lo hace con atención a conocimientos socialmente disponibles sobre las condiciones territoriales y las relaciones de fuerza que participan de su ordenamiento. Es en este sentido, se sugiere que los participantes del presente estudio vuelven reconocibles las controversias sobre el agua, empleando lo que se ha denominado "geografía política del sentido común". Dicha forma de conocimiento profano en acción implica el empleo de categorías, descriptores y predicados coherentes con el tema de discusión; pero, además, supone la constitución misma del tema en cuestión
(Shegloff, 1972). Al hablar de "España", "Cataluña" y "Valencia", los participantes en este estudio abordan los "problemas del agua" como fenómenos que trascienden el estado de cosas dado por las condiciones ambientales y los dibujan como escenarios de enfrentamientos y alianzas entre grupos con determinados intereses. Así pues, las identidades espaciales aparecen como herramientas discursivas con las que las personas co-producen los "problemas del agua" como asuntos socioespacialmente conflictivos.

La identificación de esta geografía política del sentido común puede abrir la oportunidad para al menos tres agendas de investigación futura. La primera de ellas consiste en avanzar en la comprensión social y discursiva de la identidad espacial en la interpretación que las personas hacen de las controversias tecnocientíficas públicas. La metodología empleada en este artículo -u otras como las utilizadas desde la psicología discursiva (Dixon \& Durrheim, 2000) o el análisis narrativo (Taylor, 2003) - puede resultar de suma utilidad para comprender las identidades espaciales como producto de lo que Valera y Pol (1994) llaman "relaciones dialogantes". El uso de un enfoque cualitativo como el que se propone ha demostrado ser de utilidad para acercarse al empleo que las personas hacen de las categorías identitarias y a su significado en contexto de intercambios sobre temas de interés común. De esta manera puede ofrecer un punto de vista más cercano al sentido que los protagonistas dan a sus discursos y a sus realidades. Así mismo, los datos pueden desplegarse con un mayor grado de libertad, sin que los analistas definan de antemano su contenido y estructura.

En segundo lugar, la forma como los dispositivos y categorías espaciales son usados por los participantes en los grupos de discusión sugiere que tales recursos tienen una dimensión política muy marcada. Recientemente, Dixon y Durrheim (2000) han llamado la atención sobre la necesidad de profundizar en dicha dimensión de la identidad de lugar. Una posible vía para trabajar en esta dirección consiste en indagar por las conexiones entre las categorías espaciales y marcos interpretativos con los que las personas diagnostican la realidad, realizan pronós- 
ticos y motivan a otros a actuar (Gamson, Fireman \& Rytina, 1982; Show \& Benford, 1988). En este sentido, los analistas de marcos han defendido el papel central que la identidad cumple en la identificación e intervención de problemáticas sociales (Hunt, Benford \& Snow, 2001). A partir del análisis que se ha presentado aquí, es factible afirmar que la construcción del par Gobierno-consumidores asigna a los "ciudadanos" una identidad poco proclive a la movilización. Sin embargo, participa en la emergencia de "identidades colectivas politizadas" (ver, Sabucedo, Durán \& Alzate, 2010) desde las que se denuncian situaciones de pasividad, de despilfarro y de manipulación.

Finalmente, el análisis de los datos puede leerse a la luz de lo que Darier y Tàbara (2006) llaman "categorías híbridas", esto es, formas de identidad que rompen con la separación entre lo "natural" y lo "social". Los autores utilizan los Estudios Sociales de la Ciencia y la Tecnología, y particularmente la Teoría del Actor Red, para abordar la identidad nacional como un conjunto heterogéneo constituido por naciones sin estado (como Cataluña y Quebec) y las montañas que "resuenan" en su "identidad nacional". En la misma dirección ha trabajado Michael $(2000,2004)$ a partir de la generación de "nuevos nombres heterogéneos" -denominados co(a)gentes- con los que revela procesos de hibridación identitaria (por ejemplo, entre los excursionistas y los entornos naturales que visitan). Este tipo de aproximación esta empezando a utilizarse con éxito en los estudios urbanos (ver, Farías \& Bender, 2010), y podría inaugurar un nuevo "giro" en el estudio de la identidad espacial, que vaya más allá del discurso y proponga una mirada semióticomaterial del fenómeno.

\section{Referencias}

Antaki, Ch. \& Widdicombe, S. (1998). Identities in talk. London: Sage.

Altman, I. \& Low, S. (1992). Place attachment. New York: Plenum.

Aceros, J. C., Tirado, F. \& Domènech, M. (2011). Percepciones de los ciudadanos catalanes acerca de la participación del público y los expertos en las controversias sobre el agua. Papers, 96(1), 227-244.

Bilmes, J. (2009). Taxonomies are for talking: Reanalyzing a Sacks classic. Journal of Pragmatics, 41(8), 1600-1610.

Darier, E. \& Tàbara, J. D. (2006). Els objectes naturals i la identitat nacional. Les muntanyes a Catalunya i al Quebec. Papers. Revista de Sociologia, 82, 37-55.

Dixon, J. \& Durrheim, K. (2000). Displacing place identity: A discursive approach to locating self and other. British Journal of Social Psychology, 39(1), 27-44.

Drew, P. (1978). Accusations: The occasioned use of members' knowledge of 'religious geography'. Sociology, 12(1), 1-22.

Farías, I. \& Bender, T. (2010). Urban assemblages: How Actor Network-Theory changes urban studies. New York: Routledge.

Fried, M. (1963). Grieving for a lost home. En L. J. Du$\mathrm{hl}$ (Ed.), The urban condition: People and policy in the metropolis (pp. 151-171). New Work: Simon \& Schuster.

Gamson, W. A., Fireman, B. \& Rytina, S. (1982). Encounters with unjust authority. Homewood, IL: Dorsey.

Hidalgo, M. C. \& Hernández, B. (2001). Place attachment: Conceptual and empirical questions. Journal of Environmental Psychology, 21(3), 273-281.

Housley, W. \& Fitzgerald, R. (2002). The reconsidered model of membership categorisation. Qualitative Research, 2(1), 59-74.

Housley, W. \& Smith, R. J. (2011). Mundane reason, membership categorization practices and the everyday ontology of space and place in interview talk. Qualitative Research, 11(6), 698-715.

Hunt, S., Benford, R. \& Snow, D. (2001). Marcos de acción colectiva y campos de identidad en la construcción social de los movimientos. En E. Laraña \& J. Gusfield (Eds.), Los nuevos movimientos sociales. De la ideología a la identidad (pp. 221-252). Madrid: Centro de Investigaciones Sociológicas.

Jiménez, M. (2005). El impacto politico de los movimientos sociales: un estudio de la protesta ambiental en España. Madrid: Centro de Investigaciones Sociológicas. 
Korpela, K. M. (1989). Place-identity as a product of environmental self-regulation. Journal of Environmental Psychology, 9(3), 241-256.

Lalli, M. (1992). Urban related identity: Theory, measurement and empirical findings. Journal of Environmental Psychology, 12(4), 285-303.

Lewicka, M. (2008). Place attachment, place identity, and place memory: Restoring the forgotten city past. Journal of Environmental Psychology, 28(3), 209-231.

Mairal, G. (1993). Perder el pueblo. Antropología aplicada y política hidráulica. Revista de Antropología Social, 2, 185-329.

McHoul, A. W. \& Watson, D. R. (1984). Two axes for the analysis of 'commonsense' and 'formal' geographical knowledge in classroom talk. British Journal of Sociology of Education, 5(3), 281-302.

Michael, M. (2000). Reconnecting culture, technology and nature: From society to heterogeneity. London: Routledge.

Michael, M. (2004). On making data social: Heterogeneity in sociological practice. Qualitative Research, 4(1), 5-23.

Proshansky, H. M., Fabian, A. K. \& Kaminoff, R. (1983). Place-identity: Physical world socialization of the self. Journal of Environmental Psychology, 3(1), 57 83.

Puchta, C. \& Potter, J. (2004). Focus group practice. London: Sage.

Reicher, S. (1996). Poniendo en práctica la construcción de categorías. En Á. J. Gordo \& J. L. Linaza (Eds.), Psicologías, discursos y poder (pp. 353-368). Madrid: Visor.

Rollero, C. \& De Piccoli, N. (2010). Place attachment, identification and environment perception: An empirical study. Journal of Environmental Psycho$\log y, 30(2), 198-205$.

Sabucedo, J. M., Durán, M. \& Alzate, M. (2010). Identidad colectiva movilizada. Revista de Psicología Social, 25(2), 189-201.

Schegloff, E. A. (1972). Notes on a conversational practice: Formulating place. En D. N. Sudnow (Ed.),
Studies in social interaction (pp. 75-119). New York: MacMillan/The Free Press.

Schegloff, E. A. (2007). A tutorial on membership categorization. Journal of Pragmatics, 39(3), 462-482.

Show, D. A. \& Benford, R. D. (1988). Ideology, frame resonance and participant mobilization. International Social Movement Research, 1(1), 197-217.

Silverman, D. (1998). Harvey Sacks. Social science and conversation analysis. New York: Oxford University Press.

Tajfel, H. (1981). Human groups and social categories: Studies in social psychology. Cambridge: Cambridge University Press.

Taylor, S. (2003). A place for the future? Residence and continuity in women's narratives of their lives. Narrative Inquiry, 13(1), 193-215.

Turner, J. C. (1990). Redescubrir el grupo social. Madrid: Morata.

Twigger-Ross, C. L. \& Uzzell, D. L. (1996). Place and identity process. Journal of Environmental Psycho$\log y$, 16(3), 205-220.

Uzzell, D., Pol, E. \& Badenas, D. (2002). Place identification, social cohesion, and enviornmental sustainability. Environment and Behavior, 34(1), 26-53.

Valera, S. (1997). Estudio de la relación entre el espacio simbólico urbano y los procesos de identidad social. Revista de Psicología Social, 12(2), 17-30.

Valera, S. \& Guàrdià, J. (2001). Urban social identity and sustainability: Barcelona's Olympic Village. Environment and Behavior, 34(1), 54-66.

Valera, S., Guàrdia, J. \& Pol, E. (1998). A study of the symbolic aspects of space using nonquantitative techniques of analysis. Quality $\mathcal{E}$ Quantity, 32(4), 367-381.

Valera, S. \& Pol, E. (1994). El concepto de identidad social urbana: una aproximación entre la psicología social y la psicología ambiental. Anuario de Psicología, 62(3), 5-24.

Vaughn, S., Schumm, J. S. \& Sinagub, J. (1996). Focus group interviews in education and psychology. London: Sage. 
